\title{
Impact of bias current distribution on the noise figure and power saturation of a multicontact semiconductor optical amplifier
}

\section{Robert Lennox, ${ }^{1}$ Kevin Carney, ${ }^{2}$ Ramón Maldonado-Basilio, ${ }^{2}$ Severine Philippe, ${ }^{2}$}

A. Louise Bradley, ${ }^{1, *}$ and Pascal Landais²

$1_{\text {School of Physics, Trinity College Dublin, Dublin 2, Ireland }}$

2 The RINCE Institute, School of Electronic Engineering, Dublin City University, Dublin 9, Ireland

*Corresponding author: bradlel@tcd.ie

We present an experimental investigation of a multicontact semiconductor optical amplifier. This first-generation device allows for direct control of the carrier density profile along the length of the waveguide. This is used to control the device noise figure, with a minimum value of $5 \mathrm{~dB}$ observed at a gain of $15 \mathrm{~dB}$ for an optimum carrier density profile. The opposite carrier density profile results in an increase of the power saturation by $3 \mathrm{~dB}$.

Semiconductor optical amplifiers (SOAs) have been an important topic of research over the past two decades in response to the increasing demands for higher capacity placed upon optical communications networks. As network speeds increase up to the high gigabits-per-second regime, all-optical signal processing solutions be-come increasingly attractive in an effort to bypass the limits imposed by electrical-optical-electrical conversion. SOAs, with their inherent nonlinear behavior, are the ideal candidates to feature in such all-optical processing schemes [1,2]. The use of SOAs for linear amplification of C-band optical signals is still relatively limited. Erbium-doped fiber amplifiers (EDFAs) have been the preferred solution for this application, as SOAs are penalized by an innately high noise figure (NF), usually on the order of 7 to $10 \mathrm{~dB}$ [3]. SOAs possess many other advantages, including low power consumption, small footprint, wide bandwidth, and the ability to accommodate wave-length ranges beyond the scope of EDFAs, e.g., for PON applications. However, to benefit from these features, the issue of high NF must be resolved. A number of routes to achieving reduced NF have previously been explored. An improvement in NF of between 0.5 and 2:5 dB for a fiber-to-fiber $\mathrm{NF}$ of $11 \mathrm{~dB}$ is reported using a holding beam technique [4]. Another work has analyzed the effect of 
reducing the confinement factor on the noise properties of SOAs [5], with reported reductions of $\sim 1: 5 \mathrm{~dB}$ when reducing the confinement factor for components of equal active volume. Increased saturation powers have been investigated using a single electrode device with varying contact resistance along the device length [6].

Our proposed solution has the advantages of being both simple and cost effective. Most importantly, its inherent flexibility allows the SOA to act as versatile elements as will be shown in this paper. In the proposed scheme, a multielectrode structure is used, with a bias current supplied to the SOA via three separate electro-des, providing independent control of the bias current to each section of the device [7]. Bielectrode SOAs have previously been studied as interferometric devices [8]. Additionally, multielectrode reflective SOAs have been used as low-chirp remote modulators for passive optical networks [9]. However, a multielectrode SOA structure has not yet been practically realized as a low-noise or high-saturation-power device.

The NF and power saturation of the multi-contact SOA are presented at a constant bias current and therefore a constant average carrier number density $(\mathrm{N})$. The multi-contact design is used to control the $\mathrm{N}$ distribution within the structure, which can be tailored to reduce the amplified spontaneous emission (ASE) and thus reduce the overall NF (NFtotal). The current density is kept high-er in the input section to achieve a high carrier density and lower in the output section, for a low carrier density. This particular distribution of the carriers can be understood by considering the SOA as a chain of amplifiers, corresponding to virtual subsections of the device. For each subsection, $n$, there is an associated NF (NFn) and gain (Gn). NFtotal is then given by the following equation:

$$
\mathrm{NF}_{\text {total }}=\mathrm{NF}_{1}+\frac{\mathrm{NF}_{2}-1}{G_{1}}+\cdots+\frac{\mathrm{NF}_{n}-1}{G_{1} \ldots G_{n-1}}
$$

As in a chain of separate amplifiers [10], when the lowest NF components are placed at the input of the SOA, the overall NF of the device is minimized. To conform to this condition, a high $\mathrm{N}$ must be provided in the input segment to achieve a high level of population inversion, thus lowering the inversion factor and consequently the NF [11] while increasing the gain. Lowering $N$ in the output portion has the effect of reducing backward-traveling ASE, which would otherwise deplete input carriers. At this stage, the high noise of the subsections toward the output of the device provides a minor contribution to NFtotal. Conversely, increasing the saturation output power (Psat) relies on a low carrier density at the input of the device, increasing toward the output. This ensures that, as the signal propagates, more carriers are available for amplification and, consequently, the saturation power is increased. Saturation of the gain is reduced due to decreased ASE levels, owing to the asymmetric carrier density distribution.

In this Letter, we will demonstrate the control of the NF and power saturation in a single SOA, with two different current distributions for a constant total bias of $150 \mathrm{~mA}$.

The device under test is an InGaAsP/InP bulk SOA. The device length is $700 \mu \mathrm{m}$, and there are three contacts along the waveguide, as can be seen in the inset of Fig. 1 . Three separate current sources are used to independently drive each section of the SOA. Isolation between adjacent electrodes is provided by a $10 \mu \mathrm{m}$ slot, with a resistance of $300 \Omega$ between contacts. The component may be pumped electrically via separate sources or by a resistor network in conjunction with a single source. Lasing is suppressed by an angled waveguide and high-quality antireflection coatings, 
reducing the residual spectral ripple to less than $0: 2 \mathrm{~dB}$. The device is held at a constant temperature of $21^{\circ} \mathrm{C}$.

Steady-state single-pass gain and NF characterization is undertaken using a free space, cw experimental setup. All values quoted indicate single-pass gain and NF, with losses accounted for, where the modal input loss was estimated to be $\sim 3 \mathrm{~dB}$. The optical signal is provided by a tunable external cavity laser with fiberized output, which passes through a manual polarization controller before being coupled to free space. The collimated beam is then coupled to our SOA via a 0:5 NA antireflection-coated aspheric lens. Polarization is set along the TE eigenmode of the waveguide. The SOA output is collected and coupled to an optical spectrum analyzer, with resolution bandwidth of 0:06 $\mathrm{nm}$. The proportion of ASE copolarized with the input signal is measured. The NF is calculated using experimentally obtained quantities by the following formula [12]:

$$
\mathrm{NF}(\nu)=10 \log _{10} \frac{2 \rho_{\mathrm{ASE}}(\nu)}{G(\nu) h \nu},
$$

where $\rho$ ASE is the ASE power spectral density that is co-polarized with the signal, $G$ is the single-pass gain, $h$ is Planck's constant, and $v$ is the signal frequency.

To demonstrate the impact of the carrier density profile along the device, two opposite configurations will be presented. The first bias condition is labeled 90- 50-10 mA, where $90 \mathrm{~mA}$ represents the bias current for the section at the input, $50 \mathrm{~mA}$ is for the middle section, and $10 \mathrm{~mA}$ is for the output section. The results are compared with those for the opposite bias distribution, 1050-90 mA. It can be noted that the total current applied is $150 \mathrm{~mA}$ for both cases. This limit was recom-mended by the device manufacturer for these prototype devices.

The ASE spectra, collected at the output of the device, for both bias distributions are shown in Fig. 1. The ASE is significantly lower for the 90-50-10 mA bias condition compared with that for the 1050-90 mA. Firstly, this provides evidence for the control of the carrier distribution along the device, and, secondly, based on the earlier discussion, it would be expected that the configuration showing the lower ASE should correspond to a lower NF performance. Also of note is the blue shift of the peak by 20 to $1575 \mathrm{~nm}$ and the broader ASE of the 10-50-90 mA case due to band filling resulting from a higher $\mathrm{N}$ in the output.

The wavelength dependence of the single-pass gain and the NF measured with a small coupled injected signal power of $-15 \mathrm{dBm}$, are presented in Fig. 2. A maximum gain of $15 \mathrm{~dB}$ is observed at $1565 \mathrm{~nm}$ for the $90-50-10 \mathrm{~mA}$ bias condition. A slightly lower peak gain of 14:3 dB is observed at the same wavelength for the 10-50-90 mA case. This is due to the larger value of ASE, as shown in Fig. 1, which reduces the fraction of $\mathrm{N}$ contributing to the amplification. The $3 \mathrm{~dB}$ gain bandwidth is more than $50 \mathrm{~nm}$ for both carrier distributions, where the measurement on the high wavelength side was limited by the tuning range of the external cavity laser.

The NF data for both bias conditions are also shown in Fig. 2. The minimum NF occurs at $1568 \mathrm{~nm}$, slightly red-shifted relative to the gain peak. A minimum NF of $5 \pm 0.2 \mathrm{~dB}$ is measured for the $90-50-$ $10 \mathrm{~mA}$ bias condition, whereas the NF for the opposite carrier distribution is $1.5 \mathrm{~dB}$ higher, and for the 50-50-50 $\mathrm{mA}$ bias condition it is $0.4 \mathrm{~dB}$ higher. It can also be noted that the difference in the measured NF between the 90-50-10 and 10-50-90 bias distributions increases with decreasing wavelength. This corresponds to the wavelength dependence of the difference in the level of ASE 
between the two bias conditions, as observed in Fig. 1. This demonstrates that engineering the carrier profile can be used to control the NF. The reduction in the NF compares well with that achieved using other methods, such as those described in the introduction.

The low noise configuration occurs for a higher carrier density at the input, reducing toward the output. These results are consistent with the approach of considering the SOA as a chain of subamplifiers. This can be used to gain further understanding of how the NF can be con-trolled in SOAs. The minimum NF of $5 \mathrm{~dB}$ compares favourably with currently available commercial amplifiers. Greater control of the carrier density profile could be achieved by improving the electrical isolation of the sub-sections to reduce the carrier diffusion.

The impact of the engineered carrier profile on the saturation power in the multicontact device has also been investigated, and is shown in Fig. 3. The output power dependence of the gain was measured at the wavelength of $1565 \mathrm{~nm}$, corresponding to the peak gain in Fig. 2. Considering first the 90-50-10 mA configuration, the small signal gain is 14:2 dB, and a saturation power of 6:3 dBm is measured. However, when the bias distribution is reversed, the small signal gain is $13: 7 \mathrm{~dB}$. As explained earlier, the ASE affects the small gain performance by carrier consumption. It can be seen that the decrease in gain occurs at higher output powers. In this case, the saturation power is increased by $3 \mathrm{~dB}$ to $9.3 \mathrm{dBm}$. The improvement in saturation performance is also related to the ASE, as a higher level of power is required in order to supersede the effect of the ASE.

In conclusion, a first-generation multielectrode SOA has been investigated with a view to reducing the NF or increasing the saturation power. The device concept is based on engineering the carrier profile along the SOA length where the SOA is considered as a chain of subamplifiers. The results demonstrate the versatility of the multicontact SOA, in that it can function in two distinct modes. In the low noise bias configuration, a NF of $5 \mathrm{~dB}$ has been measured, yielding a value similar to many commercial EDFAs. In the reverse bias distribution, a saturation power of 9:3 dBm is obtained. This is limited by the total current supplied to the device, which was set at a relatively low value of 150 $\mathrm{mA}$ for these prototypes, but could be increased for commercial devices. Further improvement in the electrical isolation of the sections, providing more precise control of the carrier density profile, is expected to yield further improvements in performance.

The authors acknowledge Enterprise Ireland for their support under grant CFTD/06/IT/332 and the Higher Education Authority Programme for Research in Third Level Institutions (2007-2011) via the INSPIRE programme. S. Philippe acknowledges the Irish Research Council for Science, Engineering and Technology for their support.

\section{References}

1. J. Mørk, M. L. Nielsen, and T. W. Berg, Opt. Photon. News 14(7), 42 (2003).

2. R. P. Davey, D. B. Grossman, M. Rasztovitz-Wiech, D. B. Payne, D. Nesset, A. E. Kelley, A. Rafel, S. Appathurai, and S. H. Yang, J. Lightwave Technol. 27, 273 (2009).

3. J. M. Senior, Optical Fibre Communications: Principles and Practice, 3rd ed. (Prentice-Hall, 2008).

4. F. Crottini, P. Salleras, M. A. Moreno, B. Dupertuis, B. Deveaud, and R. Brenot, IEEE Photon. Technol. Lett. 17, 977 (2005).

5. R. Brenot, F. Pommereau, O. Le Gouezigou, J. Landreau, Poinet, and L. Le Gouezigou, in Optical Fiber Communications Conference and Exposition and The National Fiber Optic 
Engineers Conference Technical Digest (CD) (Optical Society of America, 2005), paper OME50.

6. S. S. Saini, J. Bowser, R. Enck, V. Luciani, P. J. S. Heim, and Dagenais, in Proceedings of the 17th Annual Meeting of the IEEE Lasers and Electro-Optics Society (IEEE, 2004), Vol. 1, pp. 102-103.

7. F. Surre and P. Landais, "A semiconductor optical amplifier with a reduced noise figure," patent GB0821602.0 (November 26, 2008) and U.S. patent application 2010/ 0134877A1 (November 25, 2009).

8. E. Moll, Y. J. Williams, K. A. Williams, R. V. Penty, and H. White, Proc. SPIE 5723, 209 (2005).

9. G. H. De Valicourt, F. Pommereau, F. Poingt, M. Lamponi, G.-H. Duan, P. Chanclou, M. A. Violas, and R. Brenot, IEEE Photon. Technol. Lett. 22, 1425 (2010).

10. H. T. Friis, Proc. IRE 32, 419 (1944).

11. K. Carney, R. Lennox, R. Maldonado-Basilio, S. Philippe, L. Bradley, and P. Landais, Electron. Lett. 46, 1288 (2010).

12. D. M. Baney, P. Gallion, and R. S. Tucker, Opt. Fiber Technol. 6, 122 (2000).

\section{Figures}

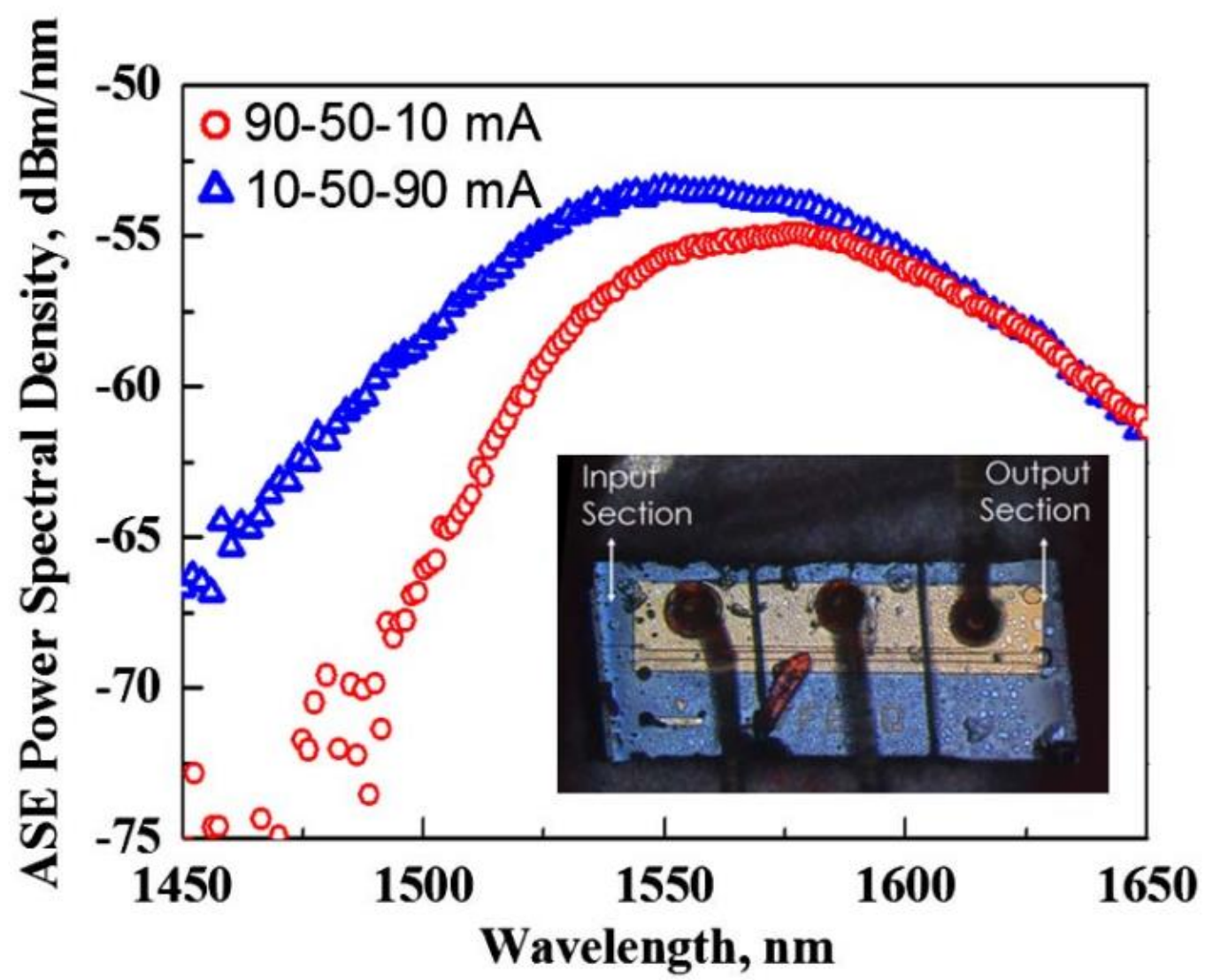

Fig. 1. ASE spectra for the two opposite bias current distributions. Inset, top-down image of SOA chip. 


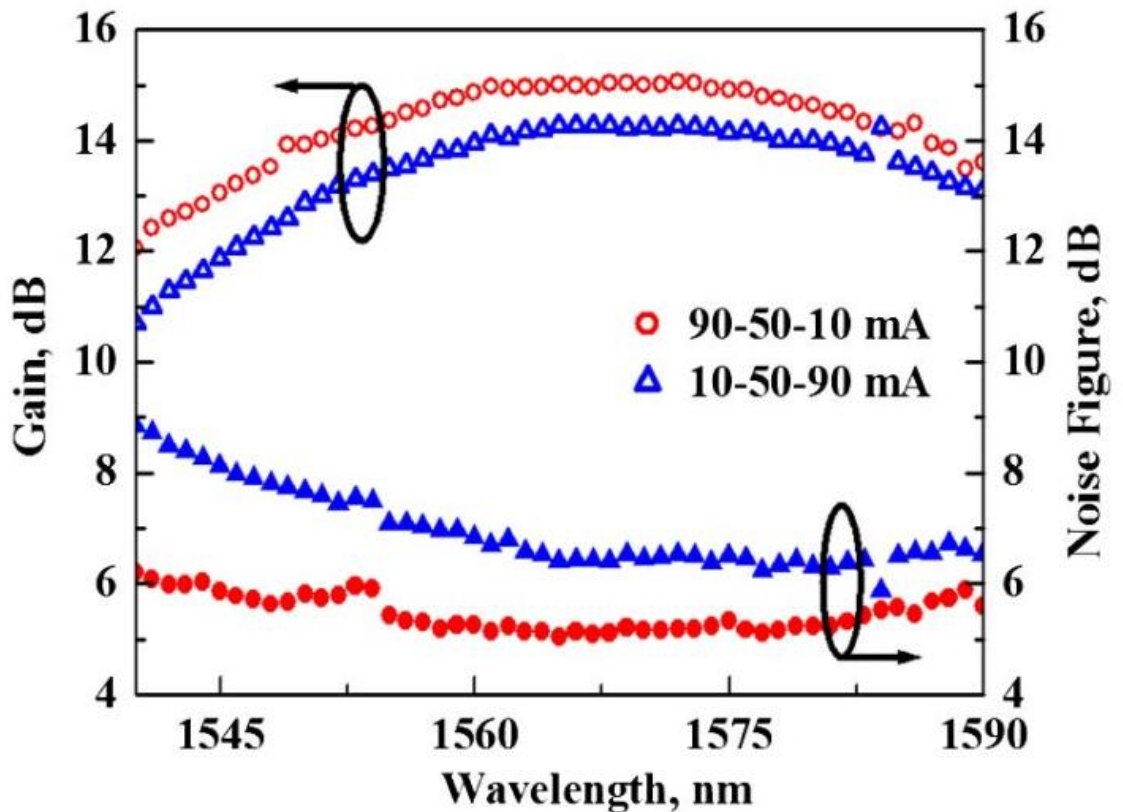

Fig. 2. Gain and NF wavelength dependence for an injected power of $-15 \mathrm{dBm}$. 


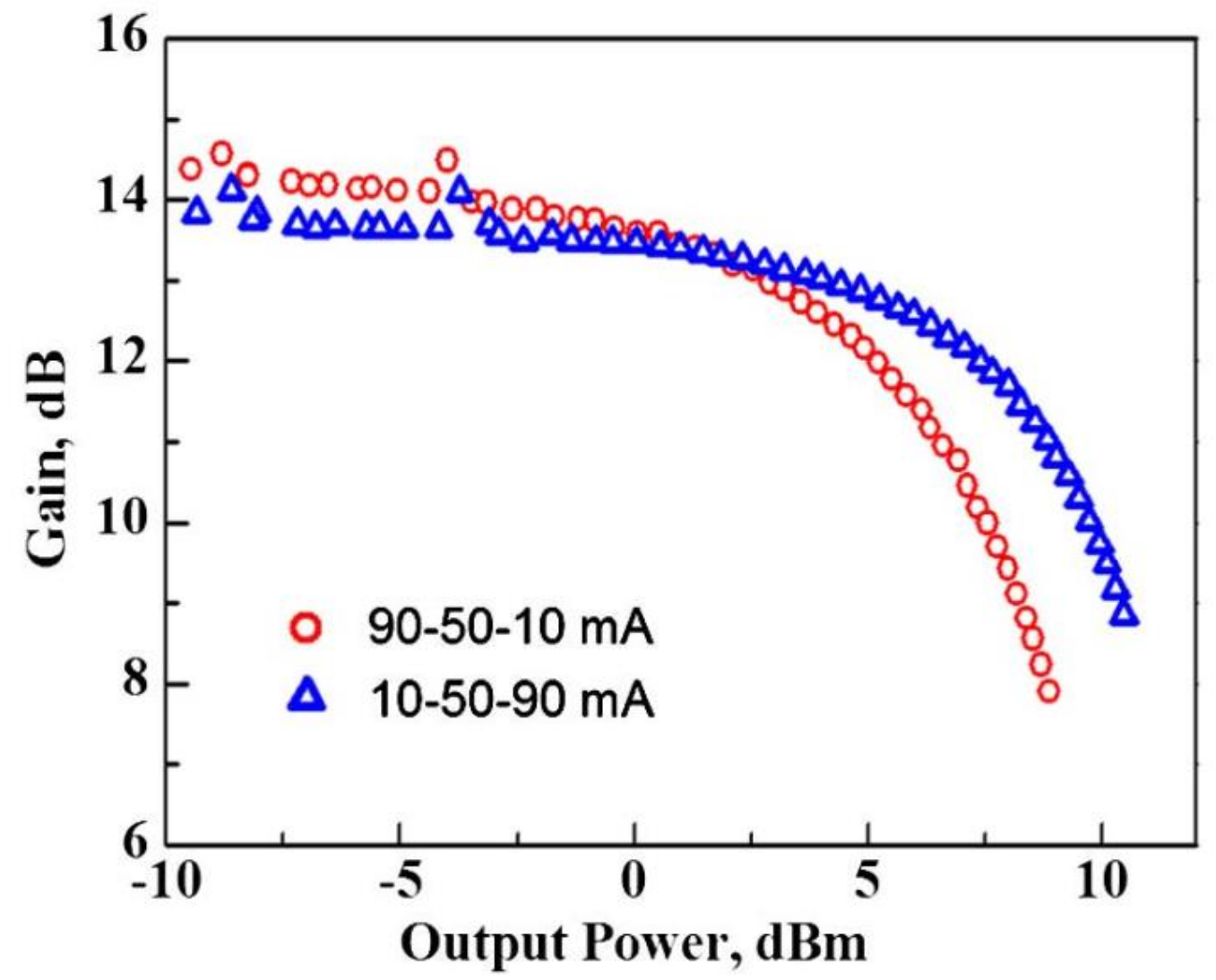

Fig. 3. Gain as a function of output power (including losses) for both current combinations, at the peak gain wavelength of $1565 \mathrm{~nm}$. 